\title{
PSYCHONOMIC SOCIETY JOURNALS Current Contents and Abstracts
}

\section{BEHAVIOR RESEARCH METHODS, INSTRUMENTS, \& COMPUTERS Volume 23, Number 1}

Calculation of additional heart rates using oxygen consumption and carbon dioxide production: $A$ comparative analysis. JULIAN F. THAYER, L. J. P. VAN DOORNEN, and J. RICK TURNER (pp. 2-4) - Research, to date, on the occurrence of additional heart rates during behavioral stressors has employed oxygen consumption as the index of metabolic activity. Although this is the obvious first choice, in certain situations measures of carbon dioxide production may be more readily obtained. The analysis presented in this paper explored the intuitively appealing notion that, since oxygen consumption and carbon dioxide production are themselves intimately related, carbon-dioxideproduction measures may also be appropriate in the "additional heart rate" methodology. Data from the most recent additionalheart-rate paper by Turner and colleagues were thus reexamined, and additional heart rates during mental arithmetic and a video game were separately calculated for the 24 subjects by first using oxygen consumption and then by using carbon dioxide production. The values obtained were very highly correlated; the coefficients for the task periods themselves were 0.99 and 0.96 , respectively. Use of carbon-dioxide-production data in this manner would, therefore, seem to be appropriate.

Extending a class of motion stimuli to study multiattribute texture perception. ANDREI GOREA and THOMAS V. PAPATHOMAS (pp. 5-8) - We present a class of stimuli that makes it possible to study the interaction of visual attributes in forming textural patterns. These stimuli are obtained in a simple manner from a class of motion stimuli that we described earlier (Papathomas \& Gorea, 1988). The main advantages of the texture stimuli presented in this paper are: (1) each attribute can be arranged simultaneously with, but independently of, other attributes, (2) an arbitrary number of attributes can be used, (3) the interaction of attributes can be studied systematically, (4) direct comparison of two attributes is possible with stimuli in which the two are arranged to form competing patterns, and (5) because of the similarity to the motion stimuli, the relationship between texture and motion mechanisms can be investigated.

Psychometric properties and correlates of three computer aversion scales. SCOTT T. MEIER and MATTHEW E. LAMBERT (pp. 9-15)-Investigators have employed a variety of constructs and measures to examine individuals' discomfort with computer use. Models and instruments for measuring constructs such as computer anxiety, computer phobia, and computer aversion have been employed to investigate negative reactions that slow individuals' acceptance and useful application of computers. Using a large college student sample, we sought to compare the psychometric properties of three computer aversion scales and to investigate their relationships to gender, age, cognitive abilities, and experience with computer use. The results indicated good reliability, good validity coefficients, and similar factor structures for the scales. Younger students, female students, and students with lower mathematical skills and less computer experience were more likely to experience discomfort with computers. Implications include the possibility that if soft- ware and hardware alter subjects' expectations about the difficulty of completing tasks, individual differences in computer aversion are likely to become a source of additional unexplained variance. Such differences must be heeded in software design and in the design of experimental research involving computers as stimulus controllers and response recorders.

A computerized infrared monitor for following movement in aquatic animals. THOMAS KIRKPATRICK, CARL W. SCHNEIDER, and RAYMOND PAVLOSKI (pp. 16-22)-Reliable monitoring of the movement of small aquatic animals presents some unique problems. In the case of the larval salamander, the animal may be less than an inch long and move in sporadic short bursts. A device is described that utilizes infrared emitters and wide-band sensors interfaced to a computer for monitoring location and movement of animals between light and dark environments over extended periods of time. The system employs a multiplexed digital input, is inexpensive, and can be easily expanded to deal with a range of monitoring problems that may occur with a wide range of both aquatic and terrestrial species.

Measuring projection tachistoscope shutter characteristics. ROBERT MADIGAN and SUSAN JOHNSON (pp. 23-26)-Projection tachistoscopes have shutters that control the presentation of stimuli. Shutter characteristics such as opening times, closing times, and mechanical reliability are important factors in applications of these tachistoscopes. The device described in this report allows the operating characteristics of shutters used in projection tachistoscopes to be evaluated. The use of the device is illustrated by measurements of three commercially available tachistoscope shutters. Each shutter was characterized by five parameters: opening and closing speeds, the lag between shutter activation and operation, the briefest stimulus presentation that the shutter allowed, and the shutter's ability to repeat ex. posure durations accurately.

Q'Hand: A fully automated apparatus for studying haptic processing of spatially distributed inputs. TOM MOORE, MICHAEL BROEKHOVEN, SUSAN LEDERMAN, and SELIM ULUG (pp. 27-35) -Q'Hand is an electromechanical system that permits fully automated preparation and presentation of spatially distributed stimulus displays, as well as on-line data acquisition, all under software control. Selected inputs may be presented to any one or combination of the middle three fin. gertips of both hands. Both accuracy and reaction times are collected on line. Q'Hand was specifically designed to investigate tactile/haptic preattentive and attentive processing of single and multidimensional stimuli. However, it may also be used to study a variety of other research topics, such as pattern recognition, psychophysical issues, and lateralization of somatosensory function. Further, it may be adapted relatively easily for use in clinical settings. For example, with this apparatus, automated psychophysical tests may be designed for the evaluation of the extent of sensory impairment due to peripheral nerve damage to the hand or to cortical dysfunction.

Automated system for ball launching, visual occlusion, and data acquisition in a ball-hitting task. KENNETH NEMIRE, MARK GOETTSCHE, and BRUCE BRIDGEMAN (pp. 36-44)Investigations of the mechanisms underlying visual-motor coor- 
dination traditionally have been limited to static situations or one-dimensional motions. Technical advances are required to determine whether experimental results from these restricted behavior domains generalize to dynamic action in three dimensions, such as ball-hitting. In this paper, we describe electromechanical devices, under computer control, for projecting a table-tennis ball, selecting monocular or binocular visual input, and recording ball-hitting performance. The automated system allows freedom of movement for the batter, can be used in a moderately sized laboratory, and can be made from easily obtainable, inexpensive parts.

Algorithms for randomness in the behavioral sciences: $A$ tutorial. MARC BRYSBAERT (pp. 45-60) -Simulations and experiments frequently demand the generation of random numbers that have specific distributions. This article describes which distributions should be used for the most common problems and gives algorithms to generate the numbers. It is also shown that a commonly used permutation algorithm (Nilsson, 1978) is deficient.

Microsecond resolution timing with Sun workstations. PETER B. DANZIG and STEVE MELVIN (pp. 61-65) -A high-resolution clock is often indispensable for the accurate measurement of millisecond or microsecond resolution intervals, and it is impossible to measure the probability distribution of short intervals without one. This paper describes a microsecond clock for Sun 3 and 4 workstations that we designed, built, and have made publicly available. It also explains how to measure intervals shorter than a computer's clock resolution, and derives confidence intervals for such measurements.

ODAP: A stand-alone program for observational data acquisition. WILLIAM P. HETRICK, ROBERT C. ISENHART, DEREK V. TAYLOR, and CURT A. SANDMAN (pp. 66-71)A software program written to collect real-time, observational data is described. The flexible program allows customized behavior codes and observational durations and simultaneously records both timed and counted events. The data are collected by means of single keystrokes, automatically stored to disk with 100 th of a second resolution and summarized for each observational session. The program's database files are dBASE III PLUS compatible and may be browsed, edited, or converted to ASCII files from the program's main menu. Field testing demonstrated the efficiency and interobserver reliability of the program (for frequency, $r=0.81$; for durational behaviors, $r s=0.89$ and 0.96). The software operates on IBM XT/AT/PS $2 \mathrm{~s}$ and most clones with PC/MS DOS version 2.0 or greater.

PSYCHOBASIC: A BASIC dialect for the control of psychological experiments with the Commodore-64 and DELA interfacing. L. XIA, C. D. L. WYNNE, F. vON MÜNCHOW-POHL, and J. D. DELIUS (pp. 72-76)-A modified version of BASIC for the control of psychological experiments is presented. PSYCHOBASIC runs on Commodore-64 computers with DELA interfacing, and it is based on Commodore BASIC. New commands control digital and analog input and output, centisecond timers, and counters. A control panel, which is displayed on the screen at all times, gives the status of many PSYCHOBASIC components. The interpreted nature of PSYCHOBASIC, as well as the control panel, makes the system easy to learn and use. The PSYCHOBASIC system is less expensive than equivalent systems for IBM-PC computers. A sample PSYCHOBASIC program is given.

CASPER: A personal computer package for exploring psychometrics. WILLIAM V. CHAMBERS and JAMES W. GRICE (pp. 79-81)-CASPER is a psychometrics software package suitable for instructional and research applications with IBMPC-compatible computers. CASPER lets the user simulate or directly enter psychometric data. Numerous statistical analyses, file handling procedures, and graphics are included. Analyses include factor analysis, multiple regression, correlation/partial correlation, moments analysis, reliability analysis, and item analysis.

Computing interrater reliability on the Apple Macintosh computer. JOHN O. BROOKS III and LAURA L. BROOKS (pp. 82 84)-A program is described for computing interrater reliability by averaging, for each rater, the correlations between one rater's ratings and every other rater's ratings. For situations in which raters rate more than one ratee, raters' reliabilities can be computed for either each item or each ratee. The program reads data from a text file and puts the reliability coefficients in a text file. The standard Macintosh interface is implemented. The QuickBASIC program is distributed both as a listing and in compiled form; it can be run with advantage with math coprocessors.

\section{Software Review}

Phasing into PHASER. JAMES T. TOWNSEND (pp. 77-78) [Differential and difference equations through computer experiments, by Hüseyin Koçak]

\section{MEMORY \& COGNITION Volume 19, Number 2}

Word repetitions in sentence recognition. KEVIN MURNANE and RICHARD M. SHIFFRIN (pp. 119-130) - When some items on a list are strengthened by extra study time or repetitions, recognition of other, unrelated, list items is not harmed (Ratcliff, Clark, \& Shiffrin, 1990). Shiffrin, Ratcliff, and Clark (1990) accounted for this list-strength finding with a model assuming that different items are stored separately in memory, but that repetitions are accumulated together into a single stronger memory trace. Repeating words in the context of different sentences might cause separate storage of the repetitions of a given word, because either word or sentence traces are stored separately. Separate storage would, in effect, convert a liststrength manipulation into a list-length manipulation and thereby induce a positive list-strength effect. In Experiment 1, this result was produced for single-word recognition and for two types of sentence recognition. In Experiment 2, both words and sentences were repeated together, which should have caused repetitions to be stored in a single, stronger, trace. As expected, the list-strength effect was eliminated. A sentence trace model was fit to the data, supporting the account of Shiffrin et al. (1990) and supporting an account of word and sentence recognition in which activation is summed for representations of all list items. The results from the two studies are inconsistent with most current models of memory (as shown by the theoretical analyses of Shiffrin et al., 1990) and pose an additional challenge for theory.

Typicality in logically defined categories: Exemplar-similarity versus rule instantiation. ROBERT M. NOSOFSKY (pp. 131150) -A rule-instantiation model and a similarity-to-exemplars model were contrasted in terms of their predictions of typicality judgments and speeded classifications for members of logically defined categories. In Experiment 1, subjects learned a unidimensional rule based on the size of objects. It was assumed that items that maximally instantiated the rule were those farthest from the category boundary that separated small and large stimuli. In Experiment 2, subjects learned a disjunctive rule of the form " $x$ or $y$ or both." It was assumed that items that maximally instantiated the rule were those with both positive values $(x$ and $y$ ). In both experiments, the frequency with which different exemplars were presented during classification learning was manipulated across conditions. These frequency manipulations 
exerted a major impact on subjects' postacquisition goodnessof-example judgments, and they also influenced reaction times in a speeded classification task. The results could not be predicted solely on the basis of the degree to which the rules were instantiated. The goodness judgments were predicted fairly well by a mixed exemplar model involving both relativesimilarity and absolute-similarity components. It was concluded that even for logically defined concepts, stored exemplars may form a major component of the category representation.

The transition from algorithm to retrieval in memory-based theories of automaticity. BRLAN J. COMPTON and GORDON D. LOGAN (pp. 151-158) -Two memory-based theories of automaticity were compared. The mixture model and the race model both describe automatization as a transition from al. gorithmic processing to memory retrieval. The mixture model predicts that, with training, the variability of reaction time will initially increase, and later decrease in a concave downward manner, whereas the race model predicts the variability will decrease only in a concave upward manner. The mixture model predicts that using both algorithm and retrieval on a single trial will be slower than using the algorithm alone, whereas the race model predicts the reverse. The experiments used an alphabet arithmetic task, in which subjects verified equations of the form $\mathrm{H}+3=\mathrm{K}$ and made subjective reports of their strategies on individual trials. Both the variability of reaction times and the pattern of reaction times associated with the strategy reports supported the race model.

The spacing effect in young children's free recall: Support for automatic-process explanations. THOMAS C. TOPPINO (pp. 159167)-The effect of spacing repetitions on children's free recall was investigated in two experiments. In Experiment 1, both 4year-old children and 7-year-old children exhibited a spacing effect in free recall, and the magnitude of the effect did not change with age. In Experiment 2, free recall was examined as a function of spacing, age ( 3 years old vs. 4 years old) and presentation rate (1 vs. 2 vs. 5 sec per stimulus). A spacing effect was obtained that did not differ as a function of age or presentation rate. Of particular interest was the fact that 3-yearolds exhibited a strong spacing effect even when stimuli were presented at a very rapid 1-sec rate. The results support the hypothesis that fundamental memory mechanisms that operate relatively automatically are sufficient to produce a spacing effect in free recall.

Incubated reminiscence effects. STEVEN M. SMITH and EDWARD VELA (pp. 168-176)-Reminiscence, the recall of material that was not successfully recalled on a previous attempt, was examined in three experiments as a function of the intertest (incubation) interval. Incubation intervals inserted between successive recall tests resulted in increased reminiscence, but the effect was seen primarily in the first retested minute. Neither the duration of the initial test (1-4 min), nor the incubation activity (maze problems vs. rest) affected this incubated reminiscence effect. The results support models in which recall tests cause output interference, but incubation intervals reduce it.

False recency and false fame of faces in young adulthood and old age. JAMES C. BARTLETT, LAURA STRATER, and ANNETTE FULTON (pp. 177-188)-Studies of age differences in face recognition have shown age-related increases in false-alarm errors: elderly persons exceed young adults in judging new faces to be old. To distinguish among theoretical accounts of this finding, we compared young and elderly subjects in two recognition tasks: (1) that of judging whether faces were recent or nonrecent, and (2) that of judging whether faces were famous or nonfamous. The major independent variable was prior presentation of faces-including nonrecent and nonfamous foils-1 week before the test. False recent judgments in response to nonrecent faces and false famous judgments in response to non. famous faces were higher among the elderly. Moreover, these age-related differences in false-alarm rates were larger for faces viewed 1 week previously than for entirely new faces. The findings suggest that, compared to young adults, older individuals rely relatively more on perceived familiarity, and relatively less on recollection of context, in making recognition decisions.

Independence of question-answering strategy and searched representation. MURRAY SINGER (pp. 189-196)-Because many studies of question answering, recall, and recognition have examined script-based messages, a spurious link between answering strategy and searched representation has emerged: Direct retrieval has been associated with the episodic representation of a discourse or a learned list, whereas plausibility judg. ment has been associated with general knowledge structures. Evidence of the independence of answering strategy and searched representation is presented. First, previous research and intuition both provide examples of the application of either strategy to either type of representation. Second, there is empirical evidence of the application of either strategy to both message text bases and general knowledge. Third, the effects of factors influencing strategy selection are independent of the type of searched representation. It is concluded that answering strategy is independent of the representation to which it is applied.

Conditions of error priming in number-fact retrieval. JAMIE I. D. CAMPBELL (pp. 197-209)-Analysis of errors in simple multiplication has shown that answers retrieved on previous trials are initially inhibited (negative error priming) but later are promoted as errors to subsequent problems (positive error priming). Two experiments investigated whether error priming is associated either with problem-specific retrieval processes or with representations of answers that can be manipulated in dependently of problems. In Experiment 1, answers were primed by visually presenting products for $200 \mathrm{msec}$ prior to problems. Correct-answer primes facilitated retrieval, related-incorrect primes interfered with retrieval more than unrelated primes, and both effects were greater for more difficult problems. Primes affected only the trial on which they were presented, however, whereas both negative and positive error priming from previous problems were observed across trials. In Experiment 2, subjects named and retrieved multiplication products on alternating trials. Just-named products were inhibited as errors to the following multiplication problem (i.e., negative error priming), but, compared to positive priming from previous retrieved products, positive error priming from previously named numbers was weak. The results indicate that positive error priming is due mainly to an encoding or retrieval bias produced by previous problems, whereas negative error priming entails suppression, or de-selection, of answer representations.

Exceptions to recognition failure as a function of the encoded association between cue and target. DAVID J. BRYANT (pp. 210219) - The relation of the recognition failure of recallable words to overall recognition rates is largely invariant across conditions that influence both recall and recognition separately. In two experiments, the influence of the integration of the mem bers of A-B word pairs on this relation was investigated. In Experiment 1 , it was found that deviations of observed recognition failure from predictions of the Tulving-Wiseman function (Tulving \& Wiseman, 1975) were produced by shallow, nonsemantic encoding. In Experiment 2, the association of categoryto-instance pairs was varied. It was found that weak associates caused larger deviations of observed recognition failure from predicted recognition failure than did strong associates. Such results suggest that a strongly encoded association between cue and target elements of A-B pairs is a necessary condition for the adherence of data to the Tulving-Wiseman function. The implications of these findings for general models of memory are discussed. 


\section{PERCEPTION \& PSYCHOPHYSICS Volume 49, Number 3}

Integration of local features as a function of global goodness and spacing. MARIA I. LASAGA and HEIKO HECHT (pp. 201211)-In two experiments, the accuracy with which subjects detected a conjunction of features was examined as a function of the spacing between items and the goodness of the axis along which they were located. In each array, two items were arranged along a vertical, a horizontal, or a diagonal axis. Based on the well-established oblique effect, the vertical and horizontal axes were considered to be good global patterns and the diagonals were considered to be poor. In Experiment 1, the two items in an array could be two horizontal lines, two vertical lines, a vertical and a horizontal line, or a plus sign with one of the single lines. In Experiment 2, a positive- and a negative-diagonal line were used as the individual features, and an " $X$ " was used as the conjunction. The results from Experiment 1 indicated that global goodness influenced only the rate of illusory conjunctions, and not of feature errors. Illusory conjunctions of vertical and horizontal line segments were more likely to occur in vertical and horizontal arrangements. The results from Experiment 2 revealed a reversal of the effect of global goodness on the rate of illusory conjunctions: Illusory conjunctions of negative- and positive-diagonal line segments were more likely to occur in diagonal arrangements. The results of both experiments taken together showed the existence of an important and new factor that influences the likelihood that features of shape will be conjoined: the ease with which line segments conjoin when they are translated along their extent toward each other. In both experiments, greater spacing between items produced more feature-identification errors and fewer feature-integration errors than did less spacing.

Visible persistence following a brief increment in stimulus luminance. CRAIG D. CLARK and JOHN H. HOGBEN (pp. 212 226) - In previous studies, it has been demonstrated that visible persistence-the period for which the perceived duration of a stimulus exceeds its physical duration-can be extended by briefly incrementing the luminance of the stimulus immediately prior to offset. Using a two-component pattern integration task, we show that this effect is not an artifact of change in the total luminous flux within the stimulus. Visible persistence was unaffected by overall luminance of the stimulus. It was also timelocked to the luminance increment. Visible persistence is seen to result from a process that is initiated by stimulus onset and that can be either wholly or partially reinitiated by the onset of the luminance increment. The duration of this process (which determines the duration of stimulus visibility) can be modified in a graded fashion by stimulus events that occur after its initiation. We outline a single-process inhibitory feedback model of the persistence mechanism that accounts for the present findings.

Simple adaptive testing with the weighted up-down method. CHRISTIAN KAERNBACH (pp. 227-229)-This paper proposes a method for adaptive testing that is less complicated than the commonly used transformed up-down methods ( 1 up 2 down, 1 up 3 down, etc.). In addition, the weighted up-down method can converge to any desired point of the psychometric function. The rule is very simple: Each correct response leads to a decrease in signal level, each incorrect response to an increase. The only difference from the simple up-down method ( 1 up 1 down) is that the steps upward and the steps downward are of a different size. The straightforward construction of the novel procedure pays off in efficiency and stability: A Monte Carlo simulation reveals a definite advantage, though small, of the weighted up-down method over the 1-up-2-down rule.

The interplay between stereopsis and structure from motion. MARK NAWROT and RANDOLPH BLAKE (pp. 230-244)-In a series of psychophysical experiments, an adaptation paradigm was employed to study the influence of stereopsis on perception of rotation in an ambiguous kinetic depth (KD) display. Without prior adaptation or stereopsis, a rotating globe undergoes spontaneous reversals in perceived direction of rotation, with successive durations of perceived rotation being random variables. Following $90 \mathrm{sec}$ of viewing a stereoscopic globe undergoing unambiguous rotation, the $K D$ globe appeared to rotate in a direction opposite that experienced during the stereoscopic adaptation period. This adaptation aftereffect was short-lived, and it occurred only when the adaptation and test figures stimulated the same retinal areas, and only when the adaptation and test figures rotated about the same axis. The aftereffect was just as strong when the test and adaptation figures had different shapes, as long as the adaptation figure contained multiple directions of motion imaged at different retinal disparities. Nonstereoscopic adaptation figures had no effect on the perceived direction of rotation of the ambiguous KD figure. These results imply that stereopsis and motion strongly interact in the specification of structure from motion, a result that complements earlier work on this problem.

Judging the relative duration of multimodal short empty time intervals. SIMON GRONDIN and ROBERT ROUSSEAU (pp. 245256) -Three experiments address the cause of the different performance levels found in time discrimination of empty intervals with durations near 250 msec. Performance differed according to the kind of sensory modality that marked the intervals. With a procedure in which the type of marker was randomized from trial to trial, it was shown that variability of discrimination judgments could not be attributed entirely to the variability of the criterion on which a judgment was based. Such a randomization slightly affects discrimination but provokes a reorganization related to marker conditions of the probabilities of judging an interval to be short or long. Moreover, it was shown that within intramodal conditions, physical characteristics of markers influence the discrimination performances. To account for the results generated with different marker-type intervals at $250 \mathrm{msec}$, we propose that two types of processor may be involved in duration discrimination: one is specifically related to a given sensory modality, whereas the other is aspecific and responsible for discrimination of intermodal intervals.

Lightness differences and the perceived segregation of regions and populations. JACOB BECK, NORMA GRAHAM, and ANNE SUTTER (pp. 257-269) - A striking finding reported by Beck, Sutter, and Ivry (1987) was that, in textures composed of regions differentiated by the arrangement (checks and stripes) of two texture elements (light and dark squares), a large lightness difference between the squares could fail to yield segregation between the regions, whereas a smaller lightness difference could sometimes yield strong segregation. In the experiments reported here, we compared the segregation of striped and checked arrangements of light and dark squares into regions with the segregation of two randomly interspersed populations of light and dark squares into subpopulations. Perceived lightnesses are the same for a given set of squares, whether they are arranged in regions or in intermixed populations. Perceived population segregation is approximately a single-valued function of the lightness differences of the squares, but perceived region segregation is not. The reason for the difference between population segregation and region segregation may be that region segregation is mediated by detectors' having large oriented receptive fields (large bar detectors) that are sensitive to the fundamental spatial frequency and orientation of the texture region as defined by the arrangement of the squares (Beck et al., 1987; Sutter, Beck, \& Graham, 1989). These detectors cannot be responsible for population segregation, because the light and dark squares are distributed randomly throughout these patterns and therefore do not define a consistent arrangement of any particular spatial frequency or orientation. The light and 
dark squares in the population patterns fall equally on excitatory and inhibitory regions of large bar detectors. A plausible explanation for population segregation is to suppose that the segregation is the result of similarity grouping of the light and dark squares.

The flanker compatibility effect as a function of visual angle, attentional focus, visual transients, and perceptual load: $A$ search for boundary conditions. JEFF MILLER (pp. 270-288)-When subjects must respond to a relevant center letter and ignore irrelevant flanking letters, the identities of the flankers produce a response compatibility effect, indicating that they are processed semantically at least to some extent. Because this effect decreases as the separation between target and flankers increases, the effect appears to result from imperfect early selection (attenuation). In the present experiments, several features of the focused attention paradigm were examined, in order to determine whether they might produce the flanker compatibility effect by interfering with the operation of an early selective mechanism. Specifically, the effect might be produced because the paradigm requires subjects to (1) attend exclusively to stimuli within a very small visual angle, (2) maintain a long-term attentional focus on a constant display location, (3) focus attention on an empty display location, (4) exclude onset-transient flankers from semantic processing, or (5) ignore some of the few stimuli in an impoverished visual field. The results indicate that none of these task features is required for semantic processing of unattended stimuli to occur. In fact, visual angle is the only one of the task features that clearly has a strong influence on the size of the flanker compatibility effect. The invariance of the flanker compatibility effect across these conditions suggests that the mechanism for early selection rarely, if ever, completely excludes unattended stimuli from semantic analysis. In addition, it shows that selective mechanisms are relatively insensitive to several factors that might be expected to influence them, thereby supporting the view that spatial separation has a special status for visual selective attention.

Semantic processing of unattended text during selective reading: How the eyes see it. ALBRECHT WERNER INHOFF and DEBORAH BRIIHL (pp. 289-294)-Subjects were instructed to read and comprehend a target (attended) passage while eye movements were recorded. A second (unattended) passage was also present, with attended and unattended passages occupying alternating lines of text. Subsequent multiple-choice questions showed acquisition of semantic information from attended and unattended text. However, a detailed examination of eyefixation records showed that readers occasionally fixated unattended text, indicating the presence of shifts of visual attention to unattended text. When fixations of unattended text were excluded, there was no longer any indication that readers obtained useful semantic information from unattended text.

Effect of imagery ability on letter-level and word-level processing. PHILIP A. ALLEN, BENJAMIN WALLACE, and ERIC WAAG (pp. 295-300)-In a letter-identification task, subjects matched a probe letter to the initial letter of a subsequently presented probe word. We varied word frequency and predicted that the performance of vivid imagers would resemble that of typical young adults, whereas the performance of poor imagers would fall in between that of typical young adults and of older adults, or would simply resemble the performance of typical older adults (a linear decrease in reaction time [RT] with word frequency; see Allen \& Madden, 1989). The in-between function for young and older adults combined predicts a dip in RT for very-high-frequency words compared to medium-high-, low-, and very-low-frequency words. As predicted, vivid imagers exhibited increased latencies for medium-high-frequency words relative to the other three word-frequency categories, whereas poor imagers exhibited a dip.

\section{PERCEPTION \& PSYCHOPHYSICS Volume 49, Number 4}

Occupancy model of perceived numerosity. JÜRI ALLIK and TIIA TUULMETS (pp. 303-314)-Observers saw 234 different pairs of stochastically organized dot patterns and indicated which of the two patterns appeared to be more numerous. All of the data can be accounted for by supposing that the choice of the more numerous pattern is based on the determination of the occupancy indices of both patterns. Each dot is posited to have an impact upon its neighborhood in a constant occupancy radius $R$. The area of the stimulus plane occupied collectively by all dots provides a basis for judging relative numerosity; the pattern with the larger occupancy value is chosen as more numerous. The occupancy model, besides providing a general explanation of known numerosity illusions in strictly quantitative terms, can explain some puzzling aspects of numerosity perception.

Recovery of 3-D shape from deforming contours. JAMES $M$. CORTESE and GEORGE J. ANDERSEN (pp. 315-327)-Three experiments were conducted to examine the accuracy of 3-D shape recovery from deforming-contour displays. The displays simulated silhouettes of ellipsoids rotating about a vertical axis. Subjects judged the horizontal crose-section of the ellipeoids. The shape of the ellipsoid, the position of the axis of rotation, and the type of projection were manipulated in Experiment 1. The results indicated relatively accurate shape recovery when the major axis of the ellipsoid was small. In Experiment 2, the shape of the ellipsoid and the velocity and curvature of the contour were manipulated. When the rate of deformation of curvature was decreased, more eccentric shapes were reported. In Experiment 3 , the shape of the object and the amount of simulated rotation were manipulated. Subjects made both shape and extent of rotation judgments. The results showed that eccentricity of shape responses could be accurately predicted from rotation responses, suggesting that the recovery of 3-D shape from smooth, deforming contours is dependent on the perceived extent of rotation.

Adaptation to form distortion of a familiar shape. ANN O'LEARY and MARGO MCMAHON (pp. 328-332)-Adaptation to the shape distortion produced by an arrangement of cylindrical lenses was demonstrated. Adaptation was produced by exposing subjects to a series of photographs of faces, which appeared horizontally stretched when seen through the lens arrangement. A 16-min adaptation procedure caused a change when the width of a test face was gradually varied and the subject indicated that it looked normal. An alteration in the perceived shape of a test face and an ellipse was also demonstrated when subjects gave estimates of the height and width of these test objects. These studies provide evidence for the operation of memory representations as the veridical cue in process assimilation.

Three-dimensional Müller-Lyer illusion. ROMI NIJHAWAN (pp. 333-341)-Three-dimensional (3-D) variants of the MüllerLyer pattern were created to address the question of where along the path of information flow in the visual system the illusion might occur. These variants, which yielded a robust illusion, included dihedral angles in place of the arrowheads of the classical pattern. The enormous difference in the shape of the resulting retinal image, compared with that of the classical pattern, makes it difficult to explain the present illusion by resorting to image-processing theories such as selective filtering (Ginsburg, 1984, 1986) or depth processing (Gregory, 1963, 1966, 1968 ). It was also shown that this $3-D$ illusion is homologous with the classical illusion, and that the two may thus share a common causal mechanism. A new type of 3-D figure, which yielded the same retinal image as did the classical pattern, was then employed. However, since the figure was 3-D, its shape 
in spatial coordinates was very different compared to that of the classical pattern. The magnitude of the illusion obtained with this figure was half that of the classical pattern. This finding suggests that the illusion might be caused by processes that occur after the computation of depth. All three experiments indicated that the illusion may be produced later in the processing stream than has previously been suggested.

Stimulus-response compatibilities between vertically oriented stimuli and horizontally oriented responses: The effects of hand position and posture. CLAIRE F. MICHAELS and SUSAN SCHILDER (pp. 342-348)-Stimulus-response (S-R) compatibility effects between vertically oriented stimuli (above or below fixation) and horizontally oriented responses (left or right switch deflections by a single hand) have been shown to depend both on which hand responds (Bauer \& Miller, 1982) and on the location at which the response is made (eccentricity on a frontoparallel line; Michaels, 1989). In the latter study, hand position and hand posture were confounded, so it is unclear which variable determined the compatibility effect. In Experiment 1, the importance of effector position was tested. Vertically oriented stimuli were paired with a horizontal response solicited at different locations but always involving the same hand posture. Compatibility effects emerged, and their direction depended on position. In Experiment 2 , the compatibilities were not evident in a simple reaction time paradigm, so the effect was not due to differential ease of responses. In Experiment 3, a change in hand posture (palm up or palm down) at the same location (the body midline) also affected the compatibilities. It was concluded that the S-R compatibility of orthogonally oriented stimuli and responses is influenced by (1) which hand responds, (2) the location of that hand, and (3) its posture. The results imply that both postural and positional states of the action system affect $S-R$ compatibility.

Moon illusion simulated in complete darkness: Planetarium experiment reexamined. KOTARO SUZUKI (pp. 349-354)-In 1962, Kaufman and Rock reported that the moon illusion did not occur in the darkness of a planetarium or in a completely dark room. The present study reexamined their findings. Two pairs of light points, separated by $3.5^{\circ}$, were presented on the dome screen of a planetarium. Subjects compared the distance between the two light points presented in the horizontal direction with the distance between the two light points at the zenith. Three illumination conditions were used: The inside of the planetarium was completely dark, was lighted, or was projected with the silhouette of a city under a starry sky. The effect of eye elevation on the illusion was also examined. Contrary to Kaufman and Rock's results, a size discrepancy comparable to the moon illusion was obtained in the horizon-and-stars condition and even in the complete-darkness condition. Little or no illusion was obtained in the lighted-room condition. The results also showed that eye elevation affected the magnitude of the illusion.

Tactile attention and the perception of moving tactile stimuli. PAUL M. EVANS and JAMES C. CRAIG (pp. 355-364)-Three experiments investigated the ability of subjects to identify the direction of movement of a pattern across the skin. In Experiments 1 and 2, subjects were required to identify the direction of movement of a pattern presented to one fingerpad while another moving pattern was being presented to an adjacent fingerpad. Subjects were instructed to attend only to the target location. The results showed that accuracy was consistently higher and reaction times were consistently faster when the two patterns moved in the same direction than when they moved in opposite directions. Both effects were largest when the two patterns were presented simultaneously. In Experiment 3, the nontarget location was the contralateral hand. In this case, performance was not affected by the presentation of the nontarget. Combined, the results suggest that movement information is processed across adjacent fingers even when subjects are explicitly instructed to attend only to one finger. Subjects do appear to be able to restrict attention to a single hand.

Identifiability of vowels and speakers from whispered syllables. VIVIEN C. TARTTER (pp. 365-372)-In the present experiments, the effect of whisper register on speech perception was measured. We assessed listeners' abilities to identify 10 vowels in [hVd] context pronounced by 3 male and 3 female speakers in normal and whisper registers. Results showed $82 \%$ average identification accuracy in whisper mode, approximately a $10 \%$ falloff in identification accuracy from normally phonated speech. In both modes, significant confusions of [0] for [a] occurred, with some additional significant confusions occurring in whisper mode among vowels adjacent in $F_{1} / F_{2}$ space. We also assessed listeners' abilities to match whispered syllables with normally phonated ones by the same speaker. Each trial contained the matching syllable and two foils whispered by speakers of the same sex as the speaker of the target. Identification performance was significantly better than chance across subjects, speakers, and vowels, with no listener achieving better than $96 \%$ performance. Acoustic analyses measured potential cues to speaker identity independent of register.

On the role of refixations in letter strings: The influence of oculomotor factors. TATJANA A. NAZIR (pp. 373-389)-Recent studies of reading and word recognition have shown that eyemovement behavior depends strongly on the position in the word that the eye first fixates; the probability of refixating in a word is lowest with the eye near the middle of the word, and it increases as the eye fixates to either side. It has generally been assumed that the cause for this optimal landing position phenomenon lies in the very strong drop-off of visual acuity even within the fovea; refixation should be more likely when the eye starts from a noncentral position, because here less information can be extracted during one fixation. It may, however, be the case that the phenomenon is caused not by acuity drop-off, but by differences in within-word oculomotor scanning tactics as a function of the position that the eye initially fixates. To test this, in the present experiment we kept visual information constant while we varied the initial fixation position. We used homogeneous strings of letters of different length. One letter in each string was different from the rest (e.g., kkkkkok), and this was the letter that the subject initially fixated. This target letter had to be identified before saccading to a comparison string. The position of the target letter in the string was varied from trial to trial. If, owing to acuity limitations, refixations reflect insufficient information extraction, then, because the target letter is always directly fixated, the pattern of refixations in this condition should be independent of the first fixation position. However, the obtained refixation probability showed a strong dependence on the position of first fixation. The num. ber of refixations was independent of the absolute length of the letter strings, but it seemed to be influenced by the proportion of the string over which the eye had to pass. The larger this proportion, the higher the probability of refixation. The results suggest that to a certain extent refixations in letter strings (or words) reflect properties of the oculomotor system rather than visual information extraction.

\section{Notes and Comment}

Perceptual constancy during ocular pursuit: A quantitative estimation procedure. S. MATEEFF, N. YAKIMOFF, J. HOHNSBEIN, and W. H. EHRENSTEIN (pp. 390-392)-Perceptual constancy of visual motion is usually described as the degree of correspondence between physical and perceived characteristics of motion in the external world. To study it, one has to assess the relationship between physical motion, its retinal image, and its perception. We describe a quantitative estimation procedure for a measure $K$ denoting the degree of perceptual constancy 
of background target motions noncollinear to the eye movements during ocular pursuit. The calculation of $K$ is based on three vectors describing the target motion (1) as it is physically, (2) as it is mapped to the retina, and (3) as it is perceived, but only the direction of the perceptual motion vector has to be determined experimentally. $K$ allows for quantitative comparison between experiments with a variety of parameters in visual motion displays.

The basis of the Bourdon illusion. PETER WENDEROTH (pp. 393-397)-Day (1990) and Day, Mitchell, and Stecher (1990) recently reported new data on the Bourdon illusion, showing that the effect occurs in novel variations of the classic figurefor example, with orthogonal and parallel test edges-and also that it occurs in the haptic modality. Day (1990) criticized theories of the Bourdon effect proposed by Wenderoth, Criss, and van der Zwan (1990), Wenderoth and O'Connor (1987a, 1987b), and Wenderoth, O'Connor, and Johnson (1986), and proposed his own "perceptual compromise" hypothesis. It is argued that Day has inaccurately portrayed the Wenderoth et al. theory, and that his own hypothesis lacks predictive power because it lacks any reference to neural mechanisms. Day's theory is thus unable to account for extant data, including his own. It is demonstrated how Day's novel experiments provide insights into the mechanisms of the Bourdon effect, and also how the Wenderoth et al. theory can account for the new data of Day (1990) and Day et al. (1990).

\section{PSYCHOBIOLOGY \\ Volume 19, Number 1}

The relationship between fact and source memory: Findings from amnesic patients and normal subjects. ARTHUR P. SHIMAMURA and LARRY R. SQUIRE (pp. 1-10)-Amnesic patients and normal subjects were presented 20 obscure facts to learn, and, after a 2-h delay, were given tests of fact recall, fact recognition, and source recall. The subjects were also given a recognition memory test in which they were asked about the learning session itself (event memory test). The amnesic patients exhibited marked impairment on all tests. Impaired source recall (i.e., source amnesia) appeared to be unrelated to impaired fact or event memory. That is, patients who made source errors remembered the facts and the learning session as well as did patients who did not make source errors. In a second experiment, normal subjects were tested $1,3,6$, and 8 weeks after learning. Source recall dropped sharply after a 6- or 8-week retention interval, demonstrating that source amnesia can occur in normal subjects when they are tested long after learning. For the normal subjects, fact and source memory were correlated. Thus, in normal subjects, source amnesia can reflect the loss of fact memory, event memory, and associations between them. However, in amnesic patients, source memory impairment was unrelated to the severity of impaired fact or event memory. Source memory impairment may reflect a specific deficit in the association of facts with their contexts.

Functional dissociation of the hippocampus and prefrontal cortex in learning and memory. GORDON WINOCUR (pp. 11-20)In Experiment 1, rats with lesions to the prefrontal cortex were tested on a variable-interval delayed alternation problem, and their performance was compared with that of rats with hippocampal lesions in a previous study (Winocur, 1985). In Experiments 2 and 3 , groups of prefrontal, hippocampal, and operated control rats were administered a test of conditional discrimination learning. Prefrontal rats were impaired in learning both tasks, but their performance was not affected by temporal manipulations that challenged episodic memory. In contrast, rats with hippocampal lesions were not impaired in basic learning, but their performance on both tasks deteriorated at relatively long delays. The results confirmed the importance of the hippocampus in long-term recall of specific events and implicated the prefrontal cortex in nonepisodic learning and memory functions.

Allocentric and egocentric spatial information processing in the hippocampal formation of the behaving primate. JANET $D$. FEIGENBAUM and EDMUND T. ROLLS (pp. 21-40)-To investigate how space is represented in the primate hippocampus, the activity of single neurons was recorded in the hippocampus of behaving macaque monkeys. Neurons that responded differently according to the position on a screen in which a stimulus was shown when the monkey had to remember the stimulus and its position were analyzed for their spatial fields. By moving the monkey into different positions relative to the screen, and the screen to different positions in the room, it was possible to separate neuronal representation in egocentric coordinates (i.e., defined relative to the monkey's body axis) from representations in local allocentric coordinates (i.e., defined relative to the frame of reference provided by the screen on which the stimuli were displayed) and from representations in absolute allocentric coordinates (i.e., defined relative to absolute position in the room). It was found that $44 \%$ of the spatial neurons analyzed in these experiments responded in relation to space defined by the local frame of reference and not in relation to space defined in egocentric or in absolute allocentric coordinates. Two percent of the neurons responded in relation to the absolute position of a stimulus in the room (in absolute allocentric coordinates). Ten percent responded in relation to egocentric coordinates. Forty-four percent responded to a combination of the different coordinate systems investigated, including $29 \%$ that were shown to include an absolute allocentric component. Thus the main finding was that in the primate hippocampus many spatial cells ( $69 \%$ of those analyzed) responded in relation to allocentric coordinates, in many cases in relation to a local frame of reference and in some cases in relation to the absolute position of the stimulus in the room. In Experiment 2 , the possibility of retinotopic encoding was investigated by presenting test spots of light at different positions relative to a fixation spot, and in different blocks of trials by moving the fixation spot to different positions on the screen. It was found that very few hippocampal cells were responsive in this task, and that for the cells that did respond, the encoding was not retinotopic. These results are consistent with the scarcity of egocentric encoding cells, and the preponderance of allocentric encoding, found in Experiment 1. In Experiment 3, it was found that relatively many hippocampal neurons (17\%) responded differently according to the spatial position being fixated on the screen, in a task in which a small fixation spot appeared on each trial in a different position on the screen. This result confirms that hippocampal spatial cells do not encode spatial information using retinotopic coordinates, shows that it is sufficient for many of these hippocampal spatial neurons to respond that the monkey fixate particular positions in space, and is consistent with the finding in Experiment 1 that the preponderant type of encoding used by these hippocampal spatial neurons is allocentric.

Spatial learning deficits are not solely due to cholinergic deficits following medial septal lesions with colchicine. STANLEY BARONE, JR., KEVIN P. NANRY, WILLIAM R. MUNDY, JACQUELINE F. MCGINTY, and HUGH A. TILSON (pp. 41-50)Colchicine was infused bilaterally into the cerebrolateral ventricles $(3.75 \mu \mathrm{g} / \mathrm{side})$ or directly into the medial septum $(5 \mu \mathrm{g})$ of adult, male Fischer-344 rats $(n=48)$, and effects on behavior and cholinergic markers were determined. Rats receiving intracerebroventricular (ICV) administration of colchicine were hyperaggressive during the first week after administration and were hyperactive when tested during 60-min sessions at weekly intervals during the first 3 weeks after colchicine treatment. ICV colchicine also interfered with the acquisition of a spatial 
task in the water maze. Rats receiving colchicine directly into the medial septum were also aggressive and hyperactive, but were not impaired in the acquisition of the water-maze task. It was subsequently found that direct administration and ICV administration of colchicine both decreased the number of choline acetyltransferase (ChAT) immunoreactive cells in the medial septum by at least $50 \%$ of vehicle-treated rats and decreased ChAT enzyme activity in both the right and the left hippocampus to about $50 \%$ of control levels. However, the acetylcholinesterase (AChE) staining was more clearly decreased bilaterally in the hippocampus after ICV infusion than after direct infusion of colchicine. ICV infusion resulted in bilateral shrinkage of the lateral septum, whereas direct septal administration resulted in only unilateral damage to the lateral septum. The results of these experiments do not support the generally accepted hypothesis that spatial learning deficits seen in animals with medial septum lesions are solely due to a lesionassociated cholinergic deficit in the hippocampus.

Does classical eyeblink conditioning generate sensitization of the neural pathway of the conditional stimulus (CS+)? MAGNE ARVE FLATEN and KENNETH HUGDAHL (pp. 51-57)-The present experiment was designed to investigate the hypothesis that classical eyeblink conditioning in humans generates sensitization of the neural pathway of the reinforced conditional stimulus (CS+). The method of startle-reflex modification was used to index sensitization. Three groups $(n=20)$ received $100 \%, 50 \%$, and $10 \%$ differential reinforcement of an auditory $\mathrm{CS}+$, while the CS - was never reinforced. The reinforcer was an airpuff to the eye, presented $865 \mathrm{msec}$ after CS + onset. After 20 conditioning trials, startle-eliciting noise was presented at different intervals after onset of the CS+ and CS-, and startle-reflex facilitation was used as an index of sensitization. Differential conditioning was observed only in the group that received $100 \%$ reinforcement. Startle-reflex facilitation was observed after onset of the CS+ in the group that received $100 \%$ reinforcement, whereas no difference was observed between startle reflexes elicited during the $\mathrm{CS}+$ and $\mathrm{CS}-$ in the other groups. The results indicate that startle-reflex facilitation is caused by sensitization of a specific neural pathway, and that startle-reflex facilitation is an indicator of neural sensitization in humans.

Comparison between the effects of the benzodiazepine receptor ligands methyl beta-carboline-3-carboxylate and diazepam in two learning situations in mice. GEORGES CHAPOUTHIER, MARIE-JOSEE RAFFALLI-SEBILLE, PATRICE VENAULT, JACQUES SIMIAND, and ROBERT H. DODD (pp. 58-63)Benzodiazepines are known to induce profound amnesia in man. In this report, we show, in mice, that whereas benzodiazepine diazepam indeed impairs performance, methyl beta-carboline3-carboxylate ( $\beta$-CCM), an inverse agonist of the benzodiazepine receptor, on the contrary enhances performance. The two learning situations used were one-trial passive avoidance and multiple-trial light-dark discrimination in a T-maze. Since these effects are blocked by Ro 15-1788, a specific antagonist of the benzodiazepine receptor, they are likely to be mediated by the benzodiazepine receptor. They seem to involve action on learning rather than on memory.

Taste cues control ethanol-induced conditioned taste avoidance but not conditioned tolerance. CHRISTOPHER L. CUNNINGHAM and CHERYL L. HALLETT (pp. 64-69)-This experiment tested the ability of taste cues to serve as the conditioned stimulus (CS) for conditioned tolerance to ethanol hypothermia. Rats were exposed to a differential Pavlovian conditioning procedure in which the manual oral infusion of one flavor solution was consistently followed by injection of ethanol $(2.25 \mathrm{~g} / \mathrm{kg})$, whereas exposure to another flavor was followed by injection of saline (16 trials each). Control rats received saline after both flavors. Body temperature was recorded continuously using radio telem. etry. Ethanol-treated rats developed tolerance to ethanol's hypothermic effect and aversion for the ethanol-paired flavor. However, flavor had no effect on the expression of tolerance, indicating that taste was not an effective CS for conditioned tolerance. This outcome may represent an instance of selective association in which taste cues more readily gain control over ethanol's effect on ingestive behavior, whereas environmental cues more readily gain control over ethanol's thermal effect. These results may also bear on the hypothesized role that ethanol's thermal effect plays in ethanol self-administration and ethanol-induced conditioned taste aversion.

Chronic ethanol treatment: Effects on the activation of brain beta-endorphin system by novelty. C. DALMAZ, C. A. NETTO, C. B. OLIVEIRA NETTO, C. A. FIN, and I. LZQUIERDO (pp. 7074) - Chronic ethanol ingestion in rats abolishes novelty-induced antinociception, as measured by a tailflick method. This treatment also inhibited the posttraining facilitatory effect of naloxone and the posttraining amnesic effect of $\beta$-endorphin in a stepdown inhibitory avoidance task. Measurement of hypothalamic $\beta$ endorphin-like immunoreactivity ( $\beta$-EPLIR) shows that there is a decrease in the levels of this peptide in chronic ethanol-treated rats. Exposure to a novel situation, which causes a decrease of $\beta$-EPLIR in control (water-treated) rats, had no effect in ethanoltreated rats. Taken together, these results suggest that chronic ethanol treatment decreases hypothalamic $\beta$ endorphin levels and makes this system unresponsive to novelty.

Effects of parasympathetic denervation of the salivary glands on feeding and drinking behavior in the rat. JUAN M. J. RAMOS and AMADEO PUERTO (pp. 75-78)-The present experiment was undertaken to study the function of parasympathetic innervation of the salivary glands on food-associated drinking in the rat. Several studies have shown nonhomeostatic polydipsia and a high number of drinking responses in the presence of dry food after removal of the salivary glands. The neural mechanisms responsible for this phenomenon, however, have not been investigated. This study shows that transection of the preganglionic parasympathetic salivary fibers at the point where they traverse the middle ear in the rat led to the development of a prandial drinking pattern. As a secondary effect of prandial drinking, experimental animals ingested a large volume of water in the presence of dry food; that is, significant polydipsia was elicited. Both the increased frequency of drinking and polydipsia were reversed when the regular dry food was replaced by a wet diet. These results are discussed in relation to the possible connection between parasympathetic innerva. tion of the salivary glands and the prandial drinking pattern characteristically observed in desalivated rats.

Changes in mesolimbic dopamine may explain stress-induced anhedonia. PAUL WILLNER, KRYSTYNA GOLEMBIOWSKA, VIOLETTA KLIMEK, and RICHARD MUSCAT (pp. 79-84)The mesolimbic dopamine projection to the limbic forebrain is known to be critically involved in responsiveness to rewards. In two experiments, the consumption of palatable weak sucrose solutions by rats was reduced by chronic exposure to mild unpredictable stress. Increases in the levels of dopamine and serotonin and their metabolites were found in the limbic forebrain of stressed rats; these changes were not present in the caudate nucleus or septal area, or in the brains of meal-fed control animals. In the first experiment ( 7 weeks of stress), specific bind ing to dopamine $\mathrm{D} 2$ receptors was decreased in limbic forebrain; this change was not seen in the second experiment ( 3 weeks of stress). We discuss the possible role of these changes in mesolimbic dopamine function in the reduced sensitivity to reward that follows exposure to chronic mild stress.

The impact of naltrexone and morphine tolerance on mild shock-induced hypoalgesia. JAMES W. GRAU, MANDY K. BILES, and PAUL A. ILLICH (pp. 85-90) - We have previously 
shown that exposure to mild shock elicits a strong hypoalgesia on the tailflick test in rats. The present studies explored the role of endogenous opioids in producing this hypoalgesia. Experiment 1 evaluated the impact of five doses of naltrexone $(0,0.44$, $1.75,7$, and $28 \mathrm{mg} / \mathrm{kg}$ ); Experiment 2 looked at the impact of morphine tolerance. Both a low dose of naltrexone $(1.75 \mathrm{mg} / \mathrm{kg})$ and morphine tolerance attenuated the hypoalgesia observed 6-10 min after mild shock. By contrast, neither morphine tolerance nor a high dose of naltrexone $(28 \mathrm{mg} / \mathrm{kg}$ ) had a significant impact on the hypoalgesia observed 2 min after shock. These findings suggest that mild shock elicits both a transient nonopioid and a long-lasting opioid hypoalgesia.

Disruption of central-place foraging in the rat following lesions of the dorsomedial thalamic nucleus. GORDON B. SCHACTER, MARIA T. PHELPS, DAVID R. BRODBECK, GORDON J. MOGENSON, and WILLIAM A. ROBERTS (pp. 91.99)-The neural basis of central-place foraging was investigated in the labora- tory rat, using a six-arm radial maze. Pieces of cheddar cheese of different sizes were placed on the ends of the maze arms, and preoperative central-place foraging behavior was monitored. Then bilateral electrolytic lesions were made in the fimbria fornix (FF), amygdala (AMY), medial frontal cortex (MFC), dorsomedial thalamic nucleus (MD), and combined MFC-MD, and a comparison was made with control animals on the tendency to carry pieces of cheese to the center of the radial-arm maze. Rats with more than $77 \%$ destruction of the MD exhibited a reduction in food-carrying behavior and a concomitant increase in eating food items at the ends of arms, in comparison with control animals. Rats with less than 77\% destruction of the MD exhibited some reduction in food-carrying behavior, but they did not differ significantly from controls. Rats with lesions of the FF, AMY, and MFC did not exhibit any reduction in foodcarrying behavior. These findings suggest that the MD plays a role in adaptive food-carrying behaviors that are involved in central-place foraging. 